\title{
Relationship between alcohol-attributable disease and socioeconomic status, and the role of alcohol consumption in this relationship: a systematic review and meta-analysis
}

Lisa Jones ${ }^{1 *}$, Geoff Bates ${ }^{1}$, Ellie McCoy ${ }^{1}$ and Mark A Bellis ${ }^{2}$

\begin{abstract}
Background: Studies show that alcohol consumption appears to have a disproportionate impact on people of low socioeconomic status. Further exploration of the relationship between alcohol consumption, socioeconomic status and the development of chronic alcohol-attributable diseases is therefore important to inform the development of effective public health programmes.

Methods: We used systematic review methodology to identify published studies of the association between socioeconomic factors and mortality and morbidity for alcohol-attributable conditions. To attempt to quantify differences in the impact of alcohol consumption for each condition, stratified by SES, we (i) investigated the relationship between SES and risk of mortality or morbidity for each alcohol-attributable condition, and (ii) where, feasible explored alcohol consumption as a mediating or interacting variable in this relationship.

Results: We identified differing relationships between a range of alcohol-attributable conditions and socioeconomic indicators. Pooled analyses showed that low, relative to high socioeconomic status, was associated with an increased risk of head and neck cancer and stroke, and in individual studies, with hypertension and liver disease. Conversely, risk of female breast cancer tended to be associated with higher socioeconomic status. These findings were attenuated but held when adjusted for a number of known risk factors and other potential confounding factors. A key finding was the lack of studies that have explored the interaction between alcohol-attributable disease, socioeconomic status and alcohol use.

Conclusions: Despite some limitations to our review, we have described relationships between socioeconomic status and a range of alcohol-attributable conditions, and explored the mediating and interacting effects of alcohol consumption where feasible. However, further research is needed to better characterise the relationship between socioeconomic status alcohol consumption and alcohol-attributable disease risk so as to gain a greater understanding of the mechanisms and pathways that influence the differential risk in harm between people of low and high socioeconomic status.
\end{abstract}

Keywords: Alcohol, Socioeconomic status, Morbidity, Mortality, Systematic review

\footnotetext{
* Correspondence: I.jones1@ljmu.ac.uk

${ }^{1}$ Centre for Public Health, Faculty of Education, Health and Community,

Liverpool John Moores University, Henry Cotton Campus, Level 2, 15-21 Web-

ster Street, Liverpool L3 2ET, UK

Full list of author information is available at the end of the article
}

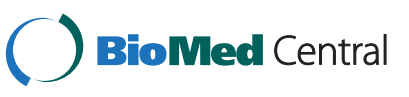

(c) 2015 Jones et al.; licensee BioMed Central. This is an Open Access article distributed under the terms of the Creative Commons Attribution License (http://creativecommons.org/licenses/by/4.0), which permits unrestricted use, distribution, and reproduction in any medium, provided the original work is properly credited. The Creative Commons Public Domain Dedication waiver (http://creativecommons.org/publicdomain/zero/1.0/) applies to the data made available in this article, unless otherwise stated. 


\section{Background}

Alcohol consumption is common in industrialised countries and globally represents the fifth largest single cause of premature mortality, loss of health and disability [1]. In 2010, alcohol use resulted in 2.7 million deaths and accounted for around $4 \%$ of global disability-adjusted life years [1]. Studies reveal a complex association between alcohol consumption and socioeconomic status (SES). While many studies have found that the burden of alcohol-related mortality and morbidity falls most heavily on people of low SES [2-7], actual alcohol consumption patterns tend to show a deviation from the traditional pattern observed with risky health behaviours. This gives rise to a paradox whereby disadvantaged populations that apparently have the same, or a lower level, of alcohol consumption suffer greater alcohol-related harm than more affluent populations.

How alcohol consumption affects the risks of health conditions has been well characterised. At lower levels of consumption, studies suggest alcohol consumption is associated with both increased health risks for some conditions (e.g. cancers, liver cirrhosis) and decreased for others (e.g. ischaemic heart disease, ischaemic stroke). It also clear that patterns of drinking, as well as volume, play an important role in both the disease burden and health benefits associated with drinking [8]. Further exploration of the relationship between alcohol consumption, SES and the development of alcohol-attributable diseases, however, is important in order to understand the contribution they make to the disproportionate impact that alcohol consumption appears to have on those of low SES and to inform the development of effective public health programmes.

We therefore used systematic review methodology to identify published studies that examined the association between socioeconomic factors and mortality and morbidity for a range of alcohol-attributable conditions. Our primary objective was to attempt to quantify the impact of alcohol consumption for each condition, stratified by SES. Our steps to achieve this objective included using meta-analysis to quantify differences in the risk of alcohol-attributable disease between high and low SES groups for studies with and without adjustment for alcohol use alone or in combination with other behaviours, and exploration of alcohol use as a mediating or interacting variable in this relationship. To our knowledge, no systematic review of this type has been undertaken previously.

\section{Methods}

The methods were based on guidelines for undertaking systematic reviews of observational studies by following the Preferred Reporting Items for Systematic reviews and Meta-analysis statement [9].

\section{Search strategy}

Searches were undertaken in Medline, Embase, PsycINFO, CINAHL, and the Web of Science in November 2012. A search strategy was developed using a combination of free text and controlled vocabulary terms and adapted for each database. See Additional file 1 for an example search strategy. As a single search strategy was used for a series of reviews on the topic of alcoholrelated harm and SES, in addition to terms for diseases partially attributable to alcohol use, the search incorporated terms for wholly attributable conditions and for injuries. References were additionally identified through searches of reference lists. The process of study selection is summarised in Figure 1.

Titles and abstracts identified through the searches were reviewed independently by two reviewers. At this stage we sought to identify studies of any alcohol-attributable condition that reported outcomes according to differing levels of SES (any measure of SES was accepted at this stage). Studies identified as potentially relevant by either reviewer were retrieved for further inspection. Full text copies of the selected studies were retrieved and independently reviewed against the full inclusion criteria by two reviewers from a team of three. Studies were retained if they met the following criteria: (i) case-control or cohort study; (ii) participants were aged 16 years or older; (iii) reported definitions and measurement of SES (including income, occupation, level of education or aggregate measures of neighbourhood-level deprivation); (iv) reported risk, odds or hazard ratios across different exposure categories of alcohol consumption; (v) reported mortality or morbidity outcomes for diseases with a known adverse risk relationship with alcohol consumption (specifically liver disease; hypertension; cancers of the mouth, head and neck, female breast, oesophagus [squamous cell carcinoma], colorectum, and liver; stroke and other cerebrovascular disorders; epilepsy; cardiac arrhythmia; and pancreatitis); (vi) published in the English language.

\section{Data extraction and quality assessment}

Methodological details recorded from studies included study details; participant details; response rates (at baseline/follow-up); follow-up duration; SES measures; and alcohol consumption measures. We also planned to extract (adjusted and unadjusted) risk estimates for each alcohol exposure category, stratified by SES. However this data was not commonly available so we extracted (adjusted and unadjusted) RRs and corresponding 95\% confidence intervals (CIs) for each alcohol exposure category and each SES category independently. For measures of SES, the adjusted risk estimates of interest were those that the study authors had attempted to control for alcohol consumption, either alone or in combination 


\section{Study selection flowchart}

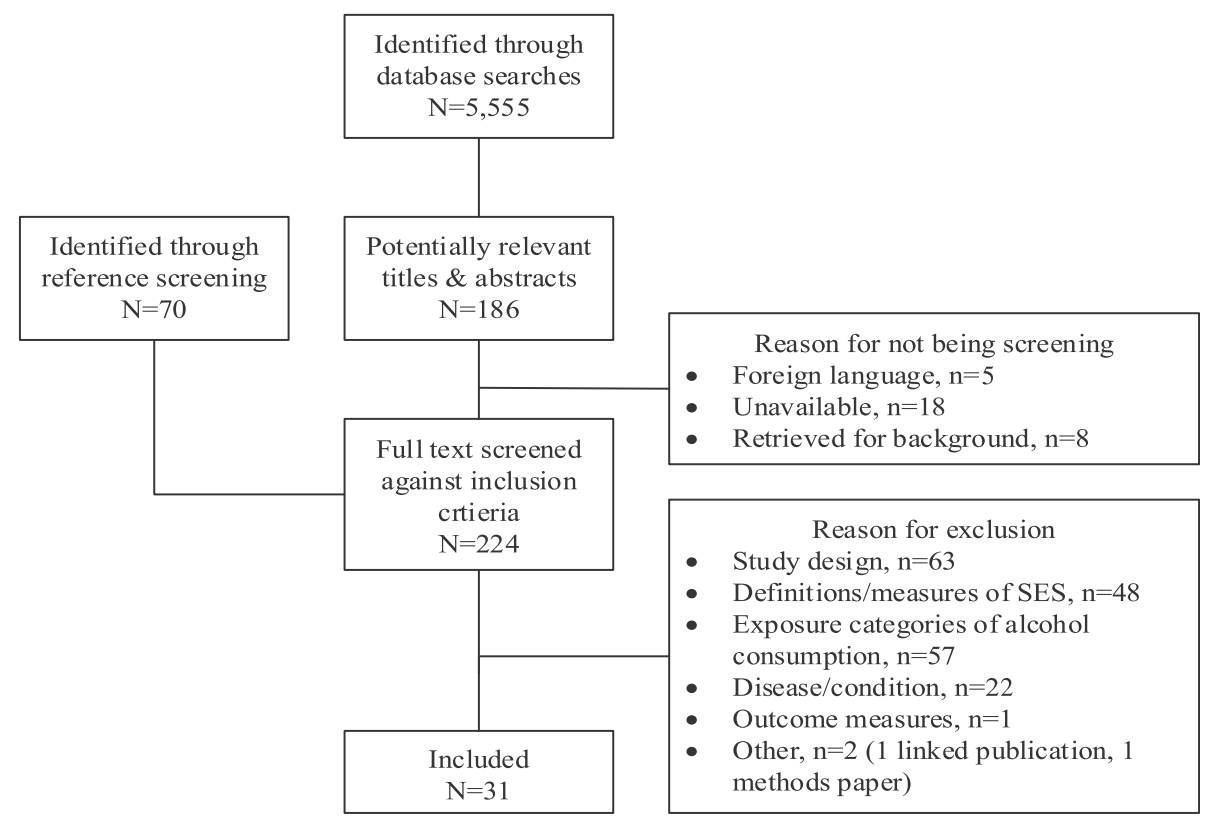

Figure 1 Study selection flowchart.

with other behavioural risk factors. The measures of SES in the included studies were reported across multiple strata. To simplify comparison across studies we retained the risk estimates comparing only the lowest and highest SES categories, using high SES as the reference category. For studies that reported low SES as the reference category, the reciprocal of the risk estimate was used to recalculate the point estimate and 95\% CIs. A small number of studies reported regression coefficients and these were extracted directly but not used in the pooled analyses. Quality was assessed using the Newcastle-Ottawa Scale. Data extraction and quality assessment were undertaken by one reviewer from a team of two and checked for accuracy by a second reviewer from a team of three.

\section{Statistical analysis}

Our planned methods included meta-analysis to generate pooled estimates of the change in risk of alcohol consumption among participants with differing SES. In practice, we identified very few studies that reported alcohol exposure categories stratified by SES. As an alternative method, we used the extracted risk estimates (OR and 95\% CI) to quantify differences in the risk of alcohol-attributable disease between high and low SES groups for studies with and without adjustment for alcohol use alone or in combination with other behaviours. As studies provided data on multiple measures of SES, to simplify the analyses and maximise data availability, we used an approach suggested by Lorant et al. [10]. Level of education was retained as the measure of SES when data on multiple measures of SES were reported, and when not available, income was considered next, followed by social occupational class and then neighbourhood level measures. We derived the log OR and corresponding standard error (SE) for each study and they were used in the pooled analyses. All meta-analyses were conducted in Review Manager (version 5.3) using the generic inverse variance outcome type. All analyses were conducted using the random effects model of DerSimonian and Laird method [11]. The $I^{2}$ statistic was used to estimate heterogeneity. We planned to assess risk of bias, specifically publication bias, through visual inspection of funnel plots. However, although funnel plots were generated, insufficient studies were included in the meta-analyses to reliably identify sources of asymmetry [12]. Forest plots were generated showing unadjusted and adjusted ORs with corresponding 95\% CIs for each study and the overall random-effects pooled estimate. Potential sources of heterogeneity were further investigated by use of visual inspection of the data, forest plots, and funnel plots.

For studies where models were adjusted for alcohol use alone we additionally calculated the percentage change in odds ratio between the highest and lowest SES category brought about by the addition of alcohol use to the unadjusted model. As a third step, we explored the 
relationship between alcohol use and SES where further analyses of the mediation or interaction between the two measures were presented.

\section{Results \\ Study identification}

The search strategy retrieved 5,555 studies and of these, 186 were selected by at least one reviewer as potentially relevant and selected for full text review. Seventy additional studies were identified through reference screening. In total, 31 papers were not screened against the full inclusion criteria; 18 papers were unavailable, 5 papers were foreign language publications and 8 papers were retrieved for background information only. A total of 224 papers were screened against the inclusion criteria and 31 studies selected for inclusion. Reasons for exclusion are summarised in the flowchart in Figure 1.

\section{Study characteristics}

Study characteristics are summarised in Table 1. Eighteen studies examined various types of cancers, six studies [13-18] examined cancers of the head and neck, three studies [19-21] examined oesophageal cancer, eight studies [22-29] examined female breast cancer, two studies $[22,28]$ examined all types of cancer, one study [28] examined smoking and alcohol-related cancers as a group, and one study [30] examined liver cancer. Seven studies [31-37] examined death or hospitalisation from stroke, two studies [38,39] systolic blood pressure (BP), and two further studies risk of hypertension $[40,41]$. Two additional studies examined atrial fibrillation [42] and liver disease [43]. Seven studies were conducted in the USA $[15,16,19,20,29,38,40]$, four in France $[17,28,39,41]$, four in the UK $[14,21,23,35]$, three in Finland $[32,34,36]$, two in Poland [18,25], two in Italy $[37,42]$ and one each in New Zealand [31], Sweden [33], Norway [22], Denmark [26], Brazil [13], Germany [24], Spain [27], South Korea [30], and Hungary [43]. Seventeen studies [13-21,24,25,27, $29,31,41-43$ ] were based on a case-control design and 14 were cohort studies [22,23,26,28,30,32-40]. Quality scores ranged from 5 to 8 out of a possible 9 . Study quality assessment is summarised in Table 2 for cohort studies and Table 3 for case-control studies. A range of SES measures were examined across the included studies including level of education [13,15-18,20,22-26,29,31,33,34,36-43], occupational social class [13,16,17,19,21,26-28,30,32,35,37], income $[19,20,23,25,26,31,38,42]$, employment status $[15,16,39]$, home ownership or tenure [38,39], community or area-level SES [14,29] and occupational mobility [28]. Fifteen studies [13,15-20,23,25,26,28,29,38,39,42] reported outcomes for more than one measure of SES. Measures of alcohol use varied across the included studies: 10 studies [15-17,19,20,25,28,29,31,34,41] reported number of drinks or glasses consumed in a day or week; 11 studies
$[13,22,24,26,27,30,32,33,37,40,42]$ reported grams or millilitres of alcohol consumed in a day, week or year; four studies $[14,21,35,43]$ reported number of units drank in a week; and one study each, reported a composite measure of number of drinks and days drank in a week [38], a dichotomous measure of drinking in the last 12 months [23]; years of vodka consumption [18]; binge drinking [36], or provided no definition [39].

It was possible to explore the relationship between alcohol-attributable disease and SES across 26 studies [13-23,25-30,32-35,37,40-43]. These findings are summarised in Table 4, which presents the extracted unadjusted and adjusted risk estimates for low compared to high SES for each of these studies. The outcomes of the pooled analyses of this relationship are shown in Table 5 and discussed further below.

\section{Alcohol-attributable cancers and SES}

Four studies [13,14,17,18] reported unadjusted outcomes for cancers of the head and neck. Pooling of these estimates revealed a statistically significant positive association between low SES and cancers of the head and neck (OR 2.72; 95\% CI 2.20, 3.37). Heterogeneity was low indicating the consistency of an increased risk among low SES groups in these studies (Figure 2). Adjustment for smoking and alcohol use was examined jointly in these studies. Pooling data across seven studies [13-18] of head and neck cancer risk showed that significance was retained after adjustment (OR 1.40; $95 \%$ CI 1.18, 1.66). Again a low level of heterogeneity indicated consistency of the findings. In general, studies found that differences in alcohol and smoking behaviours between SES groups did not fully explain the relationship between low SES and head and neck cancer. Menvielle et al. [17] noted that a substantial proportion of the risk in their study was explained by occupational exposures, but these factors were not fully considered in the other studies.

Five studies [22,23,26,28,29] reported outcomes for female breast cancer. Pooling of the unadjusted findings for these five studies showed a significant positive association between risk of breast cancer and high SES (OR 0.78 ; $95 \%$ CI $0.67,0.91$ ). However a high level of heterogeneity indicated inconsistency across the study estimates (Figure 3). Seven studies [22,23,25-29] reported odds ratios adjusted for risk factors for female breast cancer (including factors such as parity, use of HRT and BMI alongside alcohol use). Pooling of the adjusted estimates changed the direction of the effect (OR 1.04; 95\% CI $0.85,1.27)$. However, statistical tests again revealed a high level of heterogeneity and visual inspection identified the estimates from the study by Kruk et al. [25] as outliers. After exclusion of this study, heterogeneity was substantially reduced and revealed a positive, but non- 
Table 1 Characteristics of the included studies

\begin{tabular}{|c|c|c|c|c|c|c|c|c|c|c|c|}
\hline Author, Year, Country & Study years & Country & Disease area & $\begin{array}{l}\text { Study } \\
\text { type }\end{array}$ & $\begin{array}{l}\text { QA } \\
\text { score }\end{array}$ & Sex & $\begin{array}{l}\text { Age, yrs } \\
\text { (mean or range) }\end{array}$ & Cases & Controls & $\begin{array}{l}\text { SES measure(s) } \\
\text { (no. of strata) }\end{array}$ & $\begin{array}{l}\text { Alcohol measure } \\
\text { (no. of strata) }\end{array}$ \\
\hline Boing et al., 2011 [13] & 1998-2006 & Brazil & Head \& neck cancer & CC & 5 & M\&F & NR & 1,017 & 951 & $\mathrm{E}(3), \mathrm{O}(2)$ & g/yr (5) \\
\hline Braaten et al., 2005 [22] & 1991-1997 & Norway & Cancers [Various] & $\mathrm{CO}$ & 7 & $\mathrm{~F}$ & $30-69$ & 93,638 & NA & $E(4)$ & $\mathrm{g} / \mathrm{d}(4)$ \\
\hline Brown et al., 2001 [19] & 1986-1989 & USA & Oesophageal cancer & CC & 7 & M & $30-79$ & 347 & 1,354 & $E(3), O(3), I(3)$ & Drinks/wk (4) \\
\hline Brown et al., 2005 [31] & 1991-1994 \& 2003 & New Zealand & Stroke & $\mathrm{CC}$ & 6 & M\&F & 69 cases; 60 controls & 1,242 & 2,247 & I (2) & Drinks/d (2) \\
\hline Brummett et al., 2011 [38] & NR & USA & Systolic BP & $\mathrm{CO}$ & 6 & M\&F & 29 & 14,299 & NA & $E(5), I(13), T(2)$ & Days drank/wk; Drinks/wk (4) \\
\hline Chaix et al., 2010 [39] & 2007-2008 & France & Systolic BP & $\mathrm{CO}$ & 6 & M\&F & $30-79$ & 5,941 & NA & $E(4), E S(3), T(2)$ & Drinking status (4) \\
\hline Conway et al., 2010 [14] & $2002-2004$ & UK & Head \& neck cancer & CC & 7 & M\&F & NR & 103 & 91 & A (5) & Units/wk (5) \\
\hline Day et al., 1993 [15] & 1984-1985 & USA & Head \& neck cancer & CC & 5 & M\&F & $18-79$ & 1,065 & 1,182 & E (3), ES (2) & Drinks/wk (5) \\
\hline Dyer et al., 1999 [40] & 1985-1986 & USA & Hypertension & $\mathrm{CO}$ & 8 & M\&F & $18-30$ & 4,747 & NA & E (2) & $\mathrm{ml} / \mathrm{d}(\mathrm{NA})$ \\
\hline Gammon et al., 1997 [20] & 1993-1995 & USA & Oesophageal cancer & CC & 6 & M\&F & 66 & 221 & 695 & $E(6), I(5)$ & Drinks/wk (6) \\
\hline Greenberg et al., 1991 [16] & 1984-1985 & USA & Head \& neck cancer & CC & 5 & M & $18-79$ & 762 & 837 & E (3), O (3), ES (3) & Drinks/wk (5) \\
\hline Heck \& Pamuk, 1997 [23] & 1971-1975 & UK & Breast cancer & $\mathrm{CO}$ & 8 & $\mathrm{~F}$ & NR & 6,261 & NA & $E(4), I(5)$ & Last 12 months (2) \\
\hline Joshi et al., 2008 [30] & NR & South Korea & Liver cancer & $\mathrm{CO}$ & 7 & M & $30-59$ & 548,530 & NA & $\mathrm{O}(4)$ & $\mathrm{g} / \mathrm{d}(5)$ \\
\hline Kivimaki et al., 2009 [32] & $2000-2004$ & Finland & Stroke & $\mathrm{CO}$ & 7 & $\mathrm{~F}$ & 44 & 48,361 & NA & $\mathrm{O}(3)$ & g/wk (3) \\
\hline Kropp et al., 2001 [24] & 1992-1995 & Germany & Female breast cancer & CC & 7 & $\mathrm{~F}$ & 43 & 706 & 1,381 & E (3) & $\mathrm{g} / \mathrm{d}(6)$ \\
\hline Kruk et al., 2007 [25] & 2003-2007 & Poland & Female breast cancer & CC & 7 & $\mathrm{~F}$ & 55 & 858 & 1,085 & $E(4), I(3)$ & Drinks/wk (3) \\
\hline Kuper et al., 2007 [33] & 1991-1992 & Sweden & Stroke & $\mathrm{CO}$ & 8 & $\mathrm{~F}$ & 40 & 47,942 & NA & $E(4)$ & g/d (4) \\
\hline Laaksonen, et al., 2008 [34] & 1979-2001 & Finland & Stroke & $\mathrm{CO}$ & 7 & M\&F & $25-64$ & 60,608 & NA & E (3) & Drinks/wk (3) \\
\hline Larsen et al., 2011 [26] & 1993-1997 & Denmark & Female breast cancer & $\mathrm{CO}$ & 7 & $\mathrm{~F}$ & $50-64$ & 23,111 & NA & $E(3), O(7), I(4)$ & $g / d(N A)$ \\
\hline Martin-Moreno et al., 1993 [27] & 1990-1991 & Spain & Female breast cancer & CC & 7 & $\mathrm{~F}$ & $18-75$ & 762 & 988 & $\mathrm{O}(5)$ & g/d (5) \\
\hline Mattioli et al., 2006 [42] & NR & Italy & Atrial fibrillation & CC & 6 & M\&F & 54 & 116 & 116 & $E(3), I(3)$ & $\mathrm{ml} / \mathrm{d}(4)$ \\
\hline McFadden et al., 2009 [35] & 1993-1997 & UK & Stroke & $\mathrm{CO}$ & 9 & M\&F & $39-79$ & 22,488 & NA & $\mathrm{O}(5)$ & Units/wk (3) \\
\hline Melchior et al., 2005 [28] & 1989-1990 & France & Cancers [Various] & $\mathrm{CO}$ & 6 & M\&F & $35-50$ & 20,346 & NA & $\mathrm{O}(3), \mathrm{OM}$ & Glasses/d (4) \\
\hline Menvielle, et al., 2004 [17] & 1989-1991 & France & Head \& neck cancer & CC & 7 & M & NR & 504 & 242 & $\mathrm{E}(3), \mathrm{O}(3)$ & Glasses/d (6) \\
\hline Petrovski et al., 2011 [43] & 2005 & Hungary & Liver disease & CC & 5 & M & 55 cases; 54 controls & 287 & 892 & $E(4)$ & Units/wk (4); Problem drinking (2) \\
\hline Radi et al., 2005 [41] & 1997-1998 & France & Hypertension & CC & 5 & M\&F & $42 \mathrm{M} ; 44 \mathrm{~F}$ & 203 & 406 & E (3) & Glasses/d (2) \\
\hline Robert et al., 2004 [29] & 1988-1995 & USA & Female breast cancer & CC & 7 & $\mathrm{~F}$ & 62 cases; 61 controls & 7,179 & 7,488 & $E(4), A(5)$ & Drinks/d (3) \\
\hline Sharp et al., 2001 [21] & 1993-1996 & UK & Oesophageal cancer & CC & 6 & $\mathrm{~F}$ & NR & 159 & 159 & $\mathrm{O}(5)$ & Units/wk (4) \\
\hline Sundell et al., 2008 [36] & 1987, 1992 \& 1997 & Finland & Stroke & $\mathrm{CO}$ & 8 & M\&F & $25-64$ & 15,965 & NA & $E(3)$ & Binge drinking (2) \\
\hline Veronesi et al., 2010 [37] & 1986-1994 & Italy & Stroke & $\mathrm{CO}$ & 8 & M\&F & 51 & 5,084 & NA & $E(2)$ & g/d (3) \\
\hline Zatonski et al., 1991 [18] & 1986-1987 & Poland & Head \& neck cancer & CC & 7 & M & 53 cases; 44 controls & 249 & 965 & $E(3), O(3)$ & $\begin{array}{l}\text { Yrs vodka } \\
\text { consumption (4) }\end{array}$ \\
\hline
\end{tabular}


Table 2 Summary of quality assessment for cohort studies

\begin{tabular}{|c|c|c|c|c|c|c|c|c|c|c|}
\hline \multirow[t]{2}{*}{ Study, Year } & \multicolumn{4}{|c|}{ Selection } & \multicolumn{2}{|c|}{ Comparability } & \multicolumn{3}{|c|}{ Outcome } & \multirow{2}{*}{$\begin{array}{l}\text { QA } \\
\text { Score }\end{array}$} \\
\hline & 1 & 2 & 3 & 4 & $5 a$ & $5 b$ & 6 & 7 & $\overline{8}$ & \\
\hline Braaten et al., 2005 [22] & * & * & $\ldots$ & * & * & $*$ & * & * & $\ldots$ & 7 \\
\hline Brummett et al., 2011 [38] & * & * & $\ldots$ & $\cdots$ & * & * & ... & * & $\ldots$ & 5 \\
\hline Chaix et al., 2010 [39] & * & * & $\ldots$ & * & * & * & * & $\ldots$ & $\ldots$ & 6 \\
\hline Dyer et al., 1999 [40] & * & * & * & * & * & * & * & * & * & 8 \\
\hline Heck \& Palmuck, 2007 [23] & * & * & * & * & * & * & * & * & $\ldots$ & 8 \\
\hline Joshi et al., 2008 [30] & $\ldots$ & * & $\ldots$ & * & * & * & * & * & * & 7 \\
\hline Kivimaki et al., 2009 [32] & * & * & $\ldots$ & * & * & * & * & * & & 7 \\
\hline Kuper et al., 2007 [33] & * & * & $\ldots$ & * & * & * & * & * & * & 8 \\
\hline Laaksonen et al., 2007 [34] & * & * & $\ldots$ & $\ldots$ & * & * & * & * & * & 7 \\
\hline Larsen et al., 2011 [26] & * & $\ldots$ & * & * & * & * & * & * & * & 7 \\
\hline McFadden et al., 2009 [35] & * & * & * & * & * & * & * & * & & 9 \\
\hline Melchior et al., 2005 [28] & $\cdots$ & * & $\ldots$ & * & * & * & * & * & $\cdots$ & 6 \\
\hline Sundell et al., 2008 [36] & * & * & $\ldots$ & * & * & * & * & * & $*$ & 8 \\
\hline Veronesi et al., 2010 [37] & * & * & * & * & * & * & * & * & $\ldots$ & 8 \\
\hline
\end{tabular}

*, criteria met. ‥, criteria not met. Criteria: 1 , Representative of average adult in the community. 2, Drawn from same community as exposed cohort. 3, Secure record or structured interview. 4, Demonstrated. $5 \mathrm{a}=$ Yes (age, sex, alcohol \& SES). 5b = Yes (additional e.g. lifestyle factors). $6=$ Independent blind assessment, record linkage. $7=$ Follow-up $>6$ months. $8=$ Complete follow-up, or number lost $<20 \%$.

Table 3 Summary of quality assessment for case-control studies

\begin{tabular}{|c|c|c|c|c|c|c|c|c|c|c|}
\hline \multirow[t]{2}{*}{ Study, Year } & \multicolumn{4}{|c|}{ Selection } & \multicolumn{2}{|c|}{ Comparability } & \multicolumn{3}{|c|}{ Exposure } & \multirow{2}{*}{$\begin{array}{l}\text { QA } \\
\text { score }\end{array}$} \\
\hline & 1 & 2 & 3 & 4 & $5 a$ & $5 b$ & 6 & 7 & 8 & \\
\hline Boing et al., 2011 [13] & $\ldots$ & $\ldots$ & $\ldots$ & $\ldots$ & $*$ & * & $\ldots$ & * & * & 5 \\
\hline Brown et al., 2001 [19] & * & * & $\cdots$ & $\cdots$ & $*$ & * & $*$ & * & * & 7 \\
\hline Brown et al., 2005 [31] & $\ldots$ & * & * & $*$ & * & * & $\ldots$ & * & $\ldots$ & 6 \\
\hline Conway et al., 2010 [14] & * & * & * & $\ldots$ & * & * & $\cdots$ & * & * & 7 \\
\hline Day et al., 1993 [15] & $\cdots$ & $\cdots$ & * & * & * & $\ldots$ & $\cdots$ & * & * & 5 \\
\hline Gammon et al., 1997 [20] & * & * & * & * & $\ldots$ & $\ldots$ & $\ldots$ & * & * & 6 \\
\hline Greenberg et al., 1991 [16] & $\ldots$ & $\ldots$ & * & * & * & $\ldots$ & $\ldots$ & * & * & 5 \\
\hline Kropp et al., 2001 [24] & $\ldots$ & * & * & $\ldots$ & * & * & * & * & * & 7 \\
\hline Kruk et al., 2007 [25] & $\ldots$ & * & $\ldots$ & * & * & * & * & * & * & 7 \\
\hline Martin-Moreno et al., 1993 [27] & $\cdots$ & * & * & $\ldots$ & * & * & * & * & * & 7 \\
\hline Mattioli et al., 2005 [42] & * & $\ldots$ & $\ldots$ & * & * & * & * & * & $\ldots$ & 6 \\
\hline Menvielle et al., 2004 [17] & * & * & $\ldots$ & & * & * & * & * & * & 7 \\
\hline Petrovski et al., 2011 [43] & * & * & $\cdots$ & * & $\ldots$ & * & $\cdots$ & * & $\cdots$ & 5 \\
\hline Radi et al., 2005 [41] & $\ldots$ & $\ldots$ & * & * & * & * & & * & $\ldots$ & 5 \\
\hline Robert et al., 2004 [29] & $\ldots$ & * & * & * & * & * & $\ldots$ & * & * & 7 \\
\hline Sharp et al., 2001 [21] & * & * & * & $\cdots$ & $\cdots$ & * & $*$ & * & $\ldots$ & 6 \\
\hline Zatonski et al., 1991 [18] & * & * & * & $\ldots$ & $*$ & $*$ & $\ldots$ & $*$ & $*$ & 7 \\
\hline
\end{tabular}

${ }^{*}$, criteria met. ..., criteria not met. 1, Yes, independent validation. 2, Consecutive or obviously representative series of cases. 3, Community controls. 4, No history of disease (endpoint). 5a, Yes (age, sex, alcohol \& SES). 5b, Yes (additional e.g. lifestyle factors). 6, Secure record, or structured interview where blind to case/ control status. 7, Yes, same method. 8, Same rate for both groups. 
Table 4 Relationship between alcohol-attributable disease and SES

\begin{tabular}{|c|c|c|c|c|c|}
\hline \multirow[t]{2}{*}{ Study } & \multirow[t]{2}{*}{ SES measure } & \multirow[t]{2}{*}{ Sex } & \multicolumn{2}{|c|}{$\begin{array}{l}\text { RR/OR/HR }(95 \% \mathrm{Cl}) \text { for low compared to } \\
\text { high SES }\end{array}$} & \multirow[t]{2}{*}{ Variables adjusted for } \\
\hline & & & Unadjusted & Adjusted & \\
\hline \multicolumn{6}{|l|}{ Head \& neck cancer } \\
\hline Boing et al., 2011 [13] & Education & $M+F$ & $2.27(1.61,3.19)$ & $1.58(1.06,2.36)$ & Age, sex, smoking, alcohol use \\
\hline Conway et al., 2010 [14] & Neighbourhood $^{a}$ & $M+F$ & $3.62(1.35,9.71)$ & $1.90(0.59,6.09)$ & Age, sex, smoking, alcohol use \\
\hline \multirow[t]{2}{*}{ Day et al., 1993 [15] } & Education & $M+F$ & $\cdots$ & White: $1.40(1.00,1.80)$ & Smoking, alcohol use \\
\hline & & & & Black: $1.20(0.60,2.60)$ & \\
\hline Greenberg et al., 1991 [16] & Education & M & $\cdots$ & $1.0(0.7,1.5)$ & $\begin{array}{l}\text { Age, ethnicity, marital status, study area, smoking, alcohol use, snuff } \\
\text { dipping, tobacco chewing, tooth loss, denture problems, education, } \\
\text { occupational status, percentage of years worked }\end{array}$ \\
\hline Menvielle, et al., 2004 [17] & Education & M & $3.22(2.01,5.18)$ & $1.63(0.90,2.98)$ & Age, smoking, alcohol use \\
\hline Zatonski et al., 1991 [18] & Education & M & $2.94(2.03,4.27)$ & $2.51(1.06,5.94)$ & Age, smoking, alcohol use \\
\hline \multicolumn{6}{|l|}{ Female breast cancer } \\
\hline Braaten et al., 2005 [22] & Education & $\mathrm{F}$ & $0.68(0.56,0.83)$ & $0.90(0.73,1.12)$ & $\begin{array}{l}\text { Parity, alcohol use, OC, height, HRT, BMI, mammography, } \\
\text { menopausal status }\end{array}$ \\
\hline Heck \& Pamuk, 1997 [23] & Education & $\mathrm{F}$ & $0.44(0.24,0.80)$ & $0.66(0.37,1.19)$ & $\begin{array}{l}\text { Age, education, income, ethnicity, family history of breast cancer, parity, } \\
\text { age at menarche, age at menopause, OC, HRT, alcohol use, BMl, height }\end{array}$ \\
\hline \multirow[t]{2}{*}{ Kruk et al., 2007 [25] } & Education & $\mathrm{F}$ & $\cdots$ & $\begin{array}{l}\text { Pre-menopausal: } 2.39 \\
(1.58,3.60)\end{array}$ & Age, BMI, stress experience, passive smoking \\
\hline & & & & $\begin{array}{l}\text { Post-menopausal: } 1.31 \\
(0.98,1.76)\end{array}$ & \\
\hline Larsen et al., 2011 [26] & Education & $\mathrm{F}$ & $0.84(0.70,1.00)$ & $0.94(0.79,1.12)$ & HRT, parity, alcohol use, BMI \\
\hline Martin-Moreno et al., 1993 [27] & OSC & $\mathrm{F}$ & $\cdots$ & $0.63(0.33,1.17)$ & Age, study area, SES, BMI, total energy intake \\
\hline Melchior et al., 2005 [28] & OSC & $\mathrm{F}$ & $0.92(0.44,1.92)$ & $0.96(0.46,2.03)$ & $\begin{array}{l}\text { Age, smoking, alcohol use, marital status, BMI, FVC, family history of } \\
\text { breast cancer, age at first childbirth }\end{array}$ \\
\hline Robert et al., 2004 [29] & Education & $\mathrm{F}$ & $0.87(0.77,0.98)$ & $0.96(0.84,1.10)$ & $\begin{array}{l}\text { Age, interview year, community SES, urbanicity, mammography, family } \\
\text { history of breast cancer, parity, alcohol use, BMl, age at first birth, HRT, OC }\end{array}$ \\
\hline \multicolumn{6}{|l|}{ Oesophageal cancer } \\
\hline \multirow[t]{2}{*}{ Brown et al., 2001 [19] } & Education & M & $\cdots$ & White: $1.50(0.90,2.60)$ & Age, study area, alcohol use, smoking, FVC \\
\hline & & M & & Black: $3.10(1.60,6.10)$ & \\
\hline Gammon et al., 1997 [20] & Income & M & $\ldots$ & $5.00(1.67,14.99)$ & Age, sex, study area, ethnicity, BMl, smoking, alcohol use \\
\hline Sharp et al., 2001 [21] & OSC & $\mathrm{F}$ & $1.51(0.75,3.06)$ & $\cdots$ & Not applicable \\
\hline \multicolumn{6}{|l|}{ Other cancers } \\
\hline Braaten et al., 2005 [22] All cancers & Education & $\mathrm{F}$ & $0.95(0.85,1.07)$ & $\ldots$ & Not applicable \\
\hline
\end{tabular}


Table 4 Relationship between alcohol-attributable disease and SES (Continued)

\begin{tabular}{|c|c|c|c|c|c|}
\hline \multirow[t]{2}{*}{ Melchior et al., 2005 [28] All cancers } & \multirow[t]{2}{*}{ OSC } & M & $1.47(1.03,2.09)$ & $1.35(0.94,1.93)$ & \multirow{2}{*}{$\begin{array}{l}\text { Age, smoking, alcohol use, marital status, BMI, FVC, family history of } \\
\text { lung \& oral cancer }(\mathrm{M}) \text {, occupational asbestos exposure }(\mathrm{M}) \text {, family } \\
\text { history of breast cancer (F), parity (F) }\end{array}$} \\
\hline & & $\mathrm{F}$ & $1.00(0.58,1.73)$ & $1.03(0.59,1.80)$ & \\
\hline Braaten et al., 2005 [22] Colon cancer & Education & $\mathrm{F}$ & $1.23(0.70,2.18)$ & $\cdots$ & Not applicable \\
\hline Braaten et al., 2005 [22] Rectal cancer & Education & $\mathrm{F}$ & $0.63(0.33,1.21)$ & $\cdots$ & Not applicable \\
\hline Joshi et al., 2008 [30] Liver cancer & OSC & M & $1.75(1.48,2.07)$ & $1.63(1.38,1.93)$ & Age, fasting serum glucose, BMl, alcohol use, smoking \\
\hline $\begin{array}{l}\text { Melchior et al., } 2005 \text { [28] } \\
\text { Smoking/alcohol-related cancers }{ }^{b}\end{array}$ & OSC & M & $2.18(1.15,4.11)$ & $1.54(0.80,2.97)$ & $\begin{array}{l}\text { Age, smoking, alcohol use, marital status, BMI, FVC, family history of } \\
\text { lung \& oral cancer, occupational asbestos exposure }\end{array}$ \\
\hline \multicolumn{6}{|l|}{ Stroke } \\
\hline Kivimaki et al., 2009 [32] & OSC & $\mathrm{F}$ & $2.28(1.30,3.90)$ & $1.88(1.10,3.20)$ & Hypertension, CHD, diabetes, smoking, alcohol use, PA, BMI \\
\hline Kuper et al., 2007 [33] & Education & $\mathrm{F}$ & $2.10(1.40,2.90)$ & $1.80(1.30,2.60)$ & Age, alcohol use \\
\hline Laaksonen, et al., 2008 [34] & Education & $M+F$ & $1.59(0.84,2.99)$ & $1.57(0.83,2.96)$ & $\begin{array}{l}\text { Age, age squared, study year, diabetes, myocardial infarction, CHD, } \\
\text { heart failure, alcohol use }\end{array}$ \\
\hline \multirow[t]{2}{*}{ McFadden et al., 2009 [35] } & \multirow[t]{2}{*}{ OSC } & M & $2.84(1.40,5.74)$ & $2.90(1.43,5.87)$ & \multirow[t]{2}{*}{ Age, alcohol use } \\
\hline & & $\mathrm{F}$ & $2.32(1.19,4.49)$ & $2.05(1.05,4.00)$ & \\
\hline \multirow[t]{2}{*}{ Veronesi et al., 2010 [37] } & \multirow[t]{2}{*}{ Education } & M & $2.14(1.25,3.69)$ & $2.18(1.26,3.78)$ & \multirow[t]{2}{*}{ Age, hypertension, diabetes, smoking, HDL cholesterol, alcohol use } \\
\hline & & $\mathrm{F}$ & $0.54(0.26,1.12)$ & $0.40(0.20,0.85)$ & \\
\hline \multicolumn{6}{|l|}{ Hypertension } \\
\hline \multirow[t]{4}{*}{ Dyer et al., 1999 [40] } & \multirow[t]{4}{*}{ Education } & \multirow[t]{2}{*}{ M } & Black: $0.98(0.72,1.31)$ & Black: $1.01(0.72,1.41)$ & \multirow{4}{*}{$\begin{array}{l}\text { Age, systolic BP, BMl, waist circumference, PA, alcohol use, pulse, smoking, } \\
\text { education, fasting insulin, triglycerides, uric acid and } \mathrm{HDL} \text { cholesterol }\end{array}$} \\
\hline & & & White: $0.57(0.37,0.86)$ & White: $0.48(0.30,0.76)$ & \\
\hline & & \multirow[t]{2}{*}{$\mathrm{F}$} & Black: $0.65(0.47,0.89)$ & Black: $0.67(0.48,0.94)$ & \\
\hline & & & White: $0.25(0.15,0.42)$ & White: $0.42(0.23,0.78)$ & \\
\hline Radi et al., 2005 [41] & OSC & $\mathrm{F}$ & $\cdots$ & $8.12(1.30,50.73)$ & $\begin{array}{l}\text { Age, education, smoking (women), alcohol use, PA, social support at } \\
\text { work, recent stressful life events, low support outside of work (women) }\end{array}$ \\
\hline \multicolumn{6}{|l|}{ Other conditions } \\
\hline Mattioli et al., 2006 [42] Atrial fibrillation & Education & $M+F$ & $1.12(0.50,2.49)$ & $\cdots$ & Not applicable \\
\hline Petrovski et al., 2011 [43] Liver disease & Education & $M+F$ & $3.22(1.72,6.03)$ & $2.86(1.30,6.26)$ & Age, smoking, alcohol use, PA \\
\hline
\end{tabular}

${ }^{a}$ Scottish Index of Multiple Deprivation score. ${ }^{b}$ Cancers of the oral cavity and pharynx, oesophagus, pancreas, larynx, trachea \& lung, urinary tract. OR, odds ratio. RR, relative risk. HR, hazard ratio. Cl, confidence interval. $M+F$, sex data combined. OSC, occupational social class. $\cdots$, not reported. BMI, body mass index. CHD, coronary heart disease. FVC, fruit \& vegetable consumption. HR, heart rate. HRT, hormone replacement therapy. OC, oral contraceptives. PA, physical activity SES, socioeconomic status. 
Table 5 Meta-analysis results: random effects pooled risk estimates for low compared to high SES groups

\begin{tabular}{llll}
\hline Disease/Condition & Studies & Pooled risk estimate & $\mathbf{I}^{2}$ statistic \\
\hline Unadjusted & & & \\
Head and neck cancer & 4 & $2.72(2.20,3.37)$ & $0 \%$ \\
Female breast cancer & 5 & $0.78[0.67,0.91]$ & $53 \%$ \\
$\quad$ Stroke & 5 & $1.84[1.33,2.56]$ & $59 \%$ \\
Adjusted & & & \\
Head and neck cancer & 6 & $1.40[1.18,1.66]$ & $0 \%$ \\
Female breast cancer & 7 & $1.04[0.85,1.27]$ & $73 \%$ \\
Stroke & 5 & $1.65[1.13,2.41]$ & $67 \%$ \\
\hline
\end{tabular}

We used the $I^{2}$ statistic $(95 \% \mathrm{Cl})$ to estimate heterogeneity between pooled studies: $12=30-60 \%$, moderate heterogeneity; $50-90 \%$, substantial heterogeneity; $75-100 \%$, considerable heterogeneity.

significant association between risk of breast cancer and high SES (OR 0.93; 95\% CI 0.84, 1.02).

Oesophageal cancer risk was explored in three studies [19-21]. Pooling was not feasible as adjusted risk estimates were only reported for two studies. Individually these studies showed mixed findings; after adjustment for a range of lifestyle factors (including alcohol use and smoking), two studies $[19,20]$ found a statistically significant association between oesophageal cancer risk for income but not occupational social class, or level of education (data not shown). Three studies [22,28,30] examined other types of cancer but meta-analysis was not feasible as insufficient data were available. Two studies reported statistically significant associations between risk of liver cancer and low social class [30], all cancers and low social class among men and smoking and alcoholrelated cancers and low social class among men [28]. After adjustment for lifestyle factors including alcohol use, Melchior et al. [28] reported that observed gradients in risk of all cancers and smoking and alcohol-related cancers were non-significant. Joshi et al. [30] found that adjusting for alcohol use and other factors did not attenuate a significant association between SES and liver cancer risk.

\section{Alcohol-attributable cardiovascular disease and SES}

Six studies [31-35,37] reported outcomes for stroke risk. One study [31] reported regression coefficients and was not included in the meta-analysis. Pooled unadjusted data from five studies showed a positive, statistically significant relationship between low SES and stroke risk (OR 1.84; 95\% CI 1.33, 2.56). The remaining studies all reported odds ratios after adjustment for alcohol use and other conventional risk factors for stroke. Adjustment attenuated the excess risk but the pooled estimate showed that low SES remained significantly associated with risk of death or hospitalisation from stroke (OR 1.65; 95\% CI 1.13, 2.41) (Figure 4). In these five studies [31-33,35,37] assessment of heterogeneity indicated inconsistency between the adjusted and unadjusted study estimates $\left(\mathrm{I}^{2}=67 \%\right.$ and $59 \%$, respectively). Excluding the study by Veronesi et al. [37] substantially reduced the heterogeneity across both the unadjusted and adjusted pooled estimates; giving ORs of 2.14 (95\% CI: 1.71, 2.66) for the unadjusted estimates and 1.91 (95\% CI: 1.51, 2.43) for the adjusted estimates. In this study, separate estimates were presented for men and women, with the direction of the association with level of education reversed for women (i.e. lower risk associated with a low level of education) but not men. Brown et al. [31] found that lower SES was associated with a higher risk of stroke.

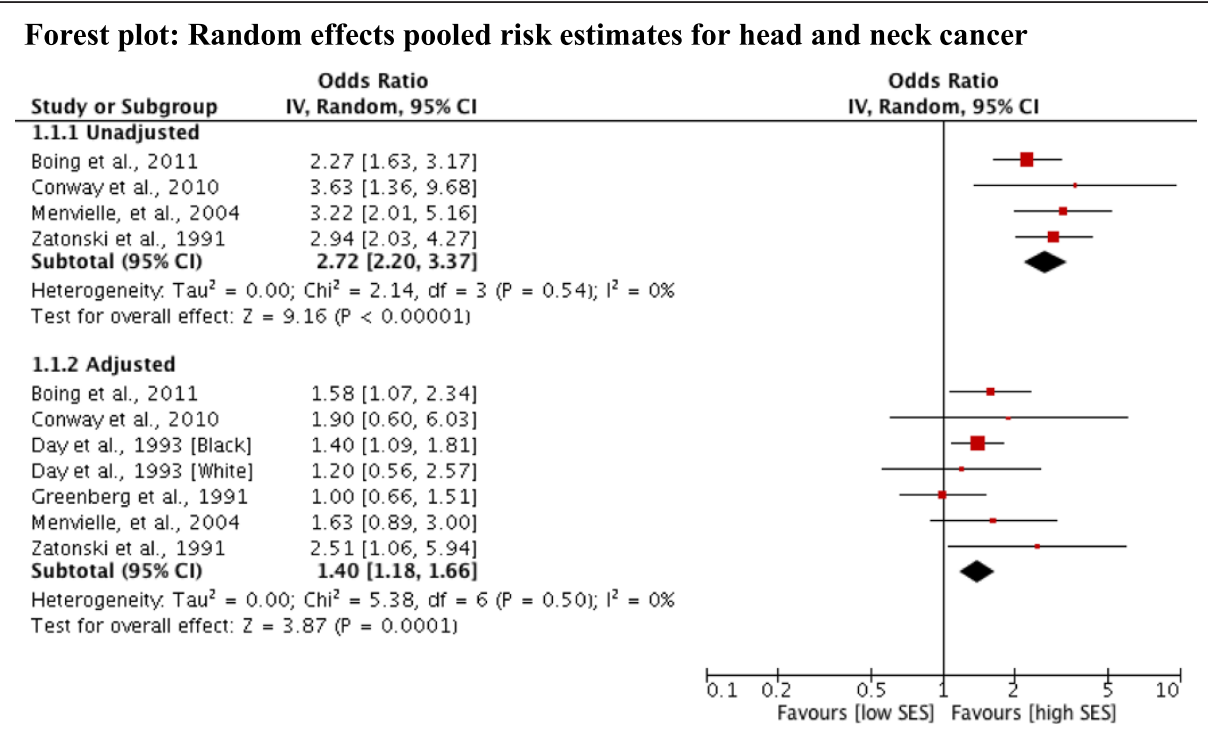

Figure 2 Forest plot: Random effects pooled risk estimates for head and neck cancer. 
Forest plot: Random effects pooled risk estimates for female breast cancer

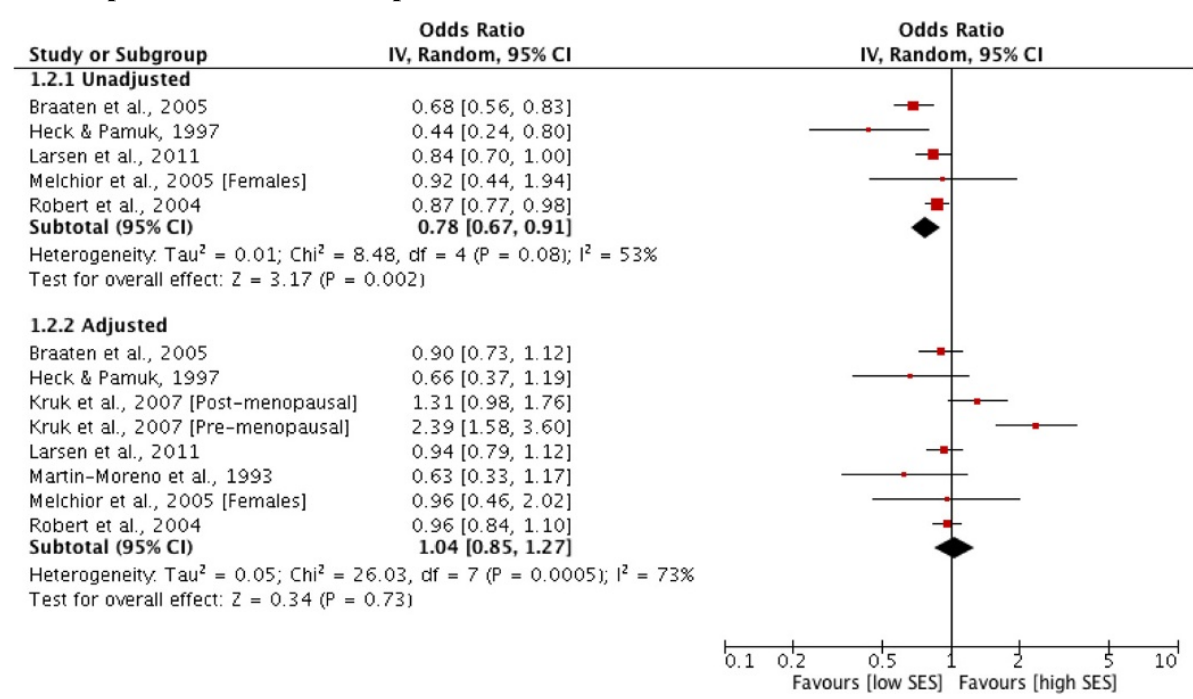

Figure 3 Forest plot: Random effects pooled risk estimates for female breast cancer.

Two studies [40,41] reported a statistically significant association between risk of hypertension and a low level of education and low level of social class, respectively. Both studies adjusted for common risk factors for hypertension (including alcohol use) and individually, for measures associated with job constraints [41] and metabolic syndrome $\mathrm{X}$ variables [40]. Two studies [38,39] reported the outcomes of an association between systolic blood pressure and SES as regression coefficients (data not shown). Both studies found an association between a low level of education and increases in systolic blood pressure, but after adjustment for behavioural and lifestyle factors such as alcohol use, an association remained only in the study by Chaix et al. [39].

\section{Other alcohol-attributable conditions and SES}

In unadjusted models, risk of liver disease was associated with a low level of education [43], whereas atrial fibrillation had no association with education or income [42]. After adjustment for behavioural factors including alcohol use, risk of liver disease remained significantly associated with a low level of education [43].

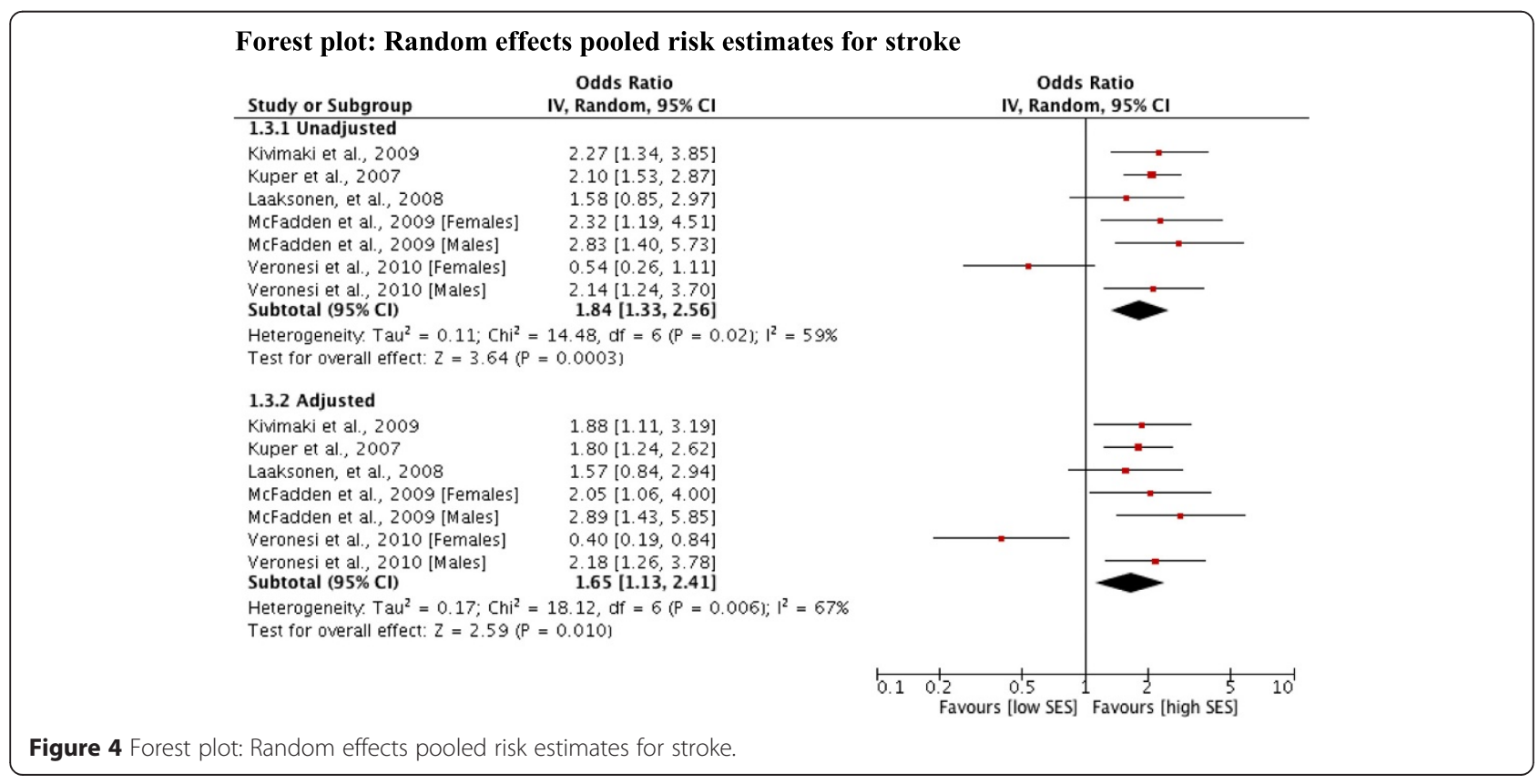




\section{Relationship between alcohol-attributable disease, SES and alcohol use}

The majority of studies included alcohol use as a potential confounder alongside other risk factors (for example, smoking, fruit and vegetable consumption, $\mathrm{BMI}$ ) and it was therefore only possible to examine the relationship between SES and alcohol use alone in two studies of stroke risk [33,35]. In addition, four studies $[13,14,17,18]$ of cancers of the head and neck reported models adjusted for alcohol use in combination with smoking.

As a mediator of the socioeconomic gradient in stroke risk, Kuper et al. [33] found that adjustment for alcohol use explained $27 \%$ and $16 \%$ of the difference in risk between high and low education groups for all strokes and ischaemic strokes, respectively. McFadden et al. [35] stratified their analyses by sex, finding that alcohol use accounted for $21 \%$ of the difference between high and low income groups among women but did not substantially decrease the odds ratio among men $(<5 \%$ difference). Low SES remained an independent predictor of stroke in adjusted models in both studies. For cancers of the head and neck, including smoking and alcohol use as covariates accounted for between 9\% [18] and 72\% [17] of the difference in risk between high and low education groups, and between $41 \%$ [17] and 61\% [18] of the difference in risk between high and low income groups. In one study [14], smoking and alcohol use in combination accounted for $66 \%$ and $71 \%$ of the difference in risk between high and low SES groups according to two separate measures of area-level deprivation. Two studies examined the role played by alcohol use as a possible mediating factor in the relationship between education and systolic blood pressure [38,39]. Alcohol use increased with education in both studies with Chaix et al. [39] noting that this finding "tended to mask rather than explain" the association between education and systolic blood pressure.
Two studies [19,24] reported SES measures stratified by alcohol consumption. Brown et al. [19] examined the combined effects of alcohol use and smoking, finding that while increasing risks were seen for each income and alcohol use category, risks were highest among heavy drinkers in the lowest income category. Kropp et al. [24] found that education status modified the effect of alcohol use on the risk of breast cancer. Risks were significantly increased among women in the highest alcohol consumption category who reported a low or intermediate level of education, compared with no significant risk among women with a high level of education. The risk estimates describing these relationships are presented in Table 6.

\section{Discussion}

Due to limitations in the data identified, the aims of this systematic review were repurposed to examine the role of alcohol consumption in the relationship between risk of alcohol-attributable disease and socioeconomic indicators, primarily level of education. The included studies covered a range of alcohol-attributable conditions, and we identified differing relationships between the selected conditions and socioeconomic indicators. Our pooled analyses showed that low, relative to high SES, was associated with an increased risk of head and neck cancer and stroke, and in individual studies, with hypertension and liver disease. Conversely, risk of female breast cancer tended to be associated with higher SES. These findings also held in models adjusted for a number of known risk factors and other potential confounding factors.

A key finding of our review is the lack of studies that have explored in depth, the relationship between alcoholattributable disease, socioeconomic status and alcohol use. In studies that adjusted for alcohol use independent of other lifestyle risk factors, its addition to statistical models explained a substantial proportion of the difference in risk between high and low SES groups for stroke risk, and in combination with smoking, head and neck cancer risk.

Table 6 Stratification of SES measures by alcohol consumption categories

\begin{tabular}{|c|c|c|c|c|c|c|}
\hline Author, Year & Disease/condition & Alcohol measure & SES measure & SES level & RR/OR/HR (95\% Cl) & Variables adjusted for \\
\hline \multirow[t]{6}{*}{ Brown et al., 2001 [19] } & \multirow[t]{6}{*}{$\begin{array}{l}\text { Oesophageal } \\
\text { cancer }\end{array}$} & \multirow[t]{3}{*}{$15-35$ drinks/wk ${ }^{a}$} & \multirow[t]{3}{*}{ Income } & Low & $71.80(15.00,343.90)$ & \multirow[t]{6}{*}{$\begin{array}{l}\text { Age, study area, raw fruit and } \\
\text { vegetable consumption, ethnicity }\end{array}$} \\
\hline & & & & Intermediate & $14.60(2.90,73.80)$ & \\
\hline & & & & High & $2.00(0.20,23.10)$ & \\
\hline & & \multirow[t]{3}{*}{$>35$ drinks $/ w^{a}$} & \multirow[t]{3}{*}{ Income } & Low & $231.60(48.20,1114.00)$ & \\
\hline & & & & Intermediate & $98.80(20.90,467.30)$ & \\
\hline & & & & High & $38.70(7.10,210.40)$ & \\
\hline \multirow[t]{3}{*}{ Kropp et al., 2001 [24] } & \multirow[t]{3}{*}{ Breast cancer } & \multirow[t]{3}{*}{$\geq 19 \mathrm{~g} / \mathrm{d}$} & \multirow[t]{3}{*}{ Education } & Low & $3.70(1.23,11.15)$ & \multirow{3}{*}{$\begin{array}{l}\text { Parity, breastfeeding, education, } \\
\text { menopausal status, family history } \\
\text { of breast cancer }\end{array}$} \\
\hline & & & & Intermediate & $1.57(1.03,2.35)$ & \\
\hline & & & & High & $0.70(0.39,1.27)$ & \\
\hline
\end{tabular}

${ }^{\mathrm{a}}$ Data presented for light smokers category only. 
Interaction was explored in two studies, of female breast cancer and oesophageal cancer risk, respectively. These studies showed that when SES measures were stratified by alcohol use, risks were greatest among low SES groups. Therefore, it may be that for some conditions, and demonstrated here for female breast cancer and hypertension, that alcohol consumption has a tendency to mask rather than explain the associations between SES and disease risk.

One of the main limitations of the review was the lack of sufficient data to conduct a meta-analysis of risk estimates stratified by SES and, therefore, our inability to fully explore the relationship between alcohol use and SES in the risk of alcohol-attributable disease. We have been unable as a consequence to estimate the overall magnitude of the association between SES, alcohol consumption, and alcohol-attributable disease risk. We are confident that our methodology was robust and comprehensive. We undertook a thorough search of the literature and retrieved a high volume of references. However, we should acknowledge the following limitations of the search; due to resource constraints we excluded articles with languages other than English and did not incorporate a search of the grey literature. Furthermore, our inclusion criteria were limited to case-control and cohort designs, and to studies of participants older than 16 years. So while in practice we found that relatively few studies presented a sufficient level of information to enable a joint analysis of SES and alcohol consumption, we acknowledge that the limitations of our search strategy and inclusion criteria may in part have contributed to the lack of study identification. It is unclear whether the lack of evidence on the interaction between SES and alcohol consumption implies that there is evidence of a lack of significant interactions for the conditions examined. Future work in this area may prove more successful if different methodological approaches are adopted, such as individual patient data meta-analysis or secondary analysis of cohorts. Further limitations in our approach may have arisen through the use of different definitions and measures of SES and alcohol consumption across the included studies, and through the presence of confounding bias. Different risk factors were adjusted for in the included studies, including by disease area, and we should therefore assume that residual confounding persists across the included studies. Our broad inclusion criteria for conditions are likely to have meant that the search strategy lacked specificity, reflected in the large volume of references retrieved. As noted, future work may benefit from taking a condition by condition approach.

\section{Conclusions}

Whilst acknowledging the scarcity of the evidence available, the findings of this review does provide further evidence that people of low SES show a greater susceptibility to the damaging effects of alcohol [44]. However, the mechanisms and pathways underlying this differential risk remain unclear and require further study. Explanatory mechanisms that have been proposed for the association between risk of alcohol-attributable disease and SES include the direct effects of: (i) differences in drinking behaviours, including quality of the alcohol consumed [45-52]; (ii) interaction through clustering of risky lifestyle behaviours, such as heavy alcohol use and smoking [53]; and (iii) differential access to healthcare [54]. Other hypothesised mechanisms include differences in the availability of social support [44] and drinking context, such as where and with whom drinking occurs [55]. Neighbourhood deprivation, acting both independently of, and in interaction with, individual SES is also thought to play a role $[56,57]$.

Despite the limitations of our review, we have described relationships between SES and a range of alcohol-attributable conditions, and explored the mediating effects of alcohol consumption where feasible. However, further research is needed to better characterise the interaction between SES, alcohol consumption and alcohol-attributable disease risk so as to gain a greater understanding of the mechanisms and pathways that influence the potentially differential risk.

\section{Additional file}

Additional file 1: Search Strategy for MEDLINE via OVID.

\section{Competing interests}

The authors declare that they have no competing interests.

\section{Authors' contributions}

$\amalg$ and MAB were responsible for the conception and design of the study. Assisted by GB, LJ coordinated the review activities including searches, study selection, data extraction and quality assessment. LJ, GB and EMC carried out the searches, and inclusion and exclusion of abstracts. $L J$ planned and led the synthesis and interpretation of data. $L J$ drafted the manuscript, and MAB, $G B$ and EMC helped with its revision. All authors read and approved the final manuscript.

\section{Acknowledgements}

The authors wish to thank our colleagues at the Centre for Public Health; in particular Professor Harry Sumnall for providing help with the revision of the manuscript. The study was funded by Alcohol Research UK as part of a flagship grant awarded to the Centre for Public Health for the research project 'Understanding the alcohol harm paradox in order to focus the development of interventions'. The funding body had no role in the design of the study, nor in the analysis of the data, or writing of the manuscript. We thank James Nicholls from Alcohol Research UK for providing advice on the interpretation of the data. We also thank Dr Nick Sheron, University of Southampton and Professor Sir lan Gilmore, University of Liverpool for their helpful advice on the interpretation of the data relating to the broader project. The corresponding author had final responsibility for the decision to submit the manuscript for publication.

\section{Author details}

${ }^{1}$ Centre for Public Health, Faculty of Education, Health and Community, Liverpool John Moores University, Henry Cotton Campus, Level 2, 15-21 
Webster Street, Liverpool L3 2ET, UK. Policy, Research and Development, Public Health Wales, Haydn Ellis Building, Maindy Road, Cardiff CF24 4HQ, UK.

\section{Received: 28 September 2014 Accepted: 30 March 2015 Published online: 18 April 2015}

\section{References}

1. Lim SS, Vos T, Flaxman AD, Danaei G, Shibuya K, Adair-Rohani H, et al. A comparative risk assessment of burden of disease and injury attributable to 67 risk factors and risk factor clusters in 21 regions, 1990-2010: a systematic analysis for the Global Burden of Disease Study 2010. Lancet. 2012;380:2224-60.

2. Erskine S, Maheswaran R, Pearson T, Gleeson D. Socioeconomic deprivation, urban-rural location and alcohol-related mortality in England and Wales. BMC Public Health. 2010;10:99.

3. Dietze PM, Jolley DJ, Chikritzhs TN, Clemens S, Catalano P, Stockwell T. Income inequality and alcohol attributable harm in Australia. BMC Public Health. 2009;9:70.

4. Castillo-Carniglia Á, Kaufman JS, Pino P. Small area associations between social context andalcohol-attributable mortality in a middle income country. Drug Alcohol Depend. 2014;137:129-36.

5. Connolly S, O'Reilly D, Rosato M, Cardwell C. Area of residence and alcohol-related mortality risk: a five-year follow-up study. Addiction. 2011;106(1):84-92.

6. Hemstrom O. Alcohol-related deaths contribute to socioeconomic differentials in mortality in Sweden. Eur J Public Health. 2002;12(4):254-62.

7. Makela P. Alcohol-related mortality as a function of socio-economic status. Addiction. 1999;94(6):867-86.

8. Babor TF, Caetano R, Casswell S, Edwards G, Giesbrecht N, Graham K, et al. Alcohol: no ordinary commodity. Research and public policy. New York: Oxford University Press; 2010.

9. Stroup DF, Berlin JA, Morton SC, Olkin I, Williamson GD, Rennie D, et al. Meta-analysis of observational studies in epidemiology: a proposal for reporting. Meta-analysis Of Observational Studies in Epidemiology (MOOSE) group. JAMA. 2000;283:2008-12.

10. Lorant V, Deliège $D$, Eaton W, Robert A, Philippot P, Ansseau M. Socioeconomic inequalities in depression: a meta-analysis. Am J Epidemiol. 2003;157:98-112.

11. DerSimonian R, Laird N. Meta-analysis in clinical trials. Control Clin Trial. 1986;7:177-88

12. Sterne J, Sutton A, loannidis J, Terrin N, Jones D, Lau J, et al. Recommendations for examining and interpreting funnel plot asymmetry in meta-analyses of randomised controlled trials. BMJ. 2011;343:d4002.

13. Boing AF, Antunes JLF, de Carvalho MB, de Gois Filho JF, Kowalski LP, Michaluart Jr P, et al. How much do smoking and alcohol consumption explain socioeconomic inequalities in head and neck cancer risk? J Epidemiol Community Health. 2011;65(8):709-14.

14. Conway DI, McMahon AD, Smith K. Components of socioeconomic risk associated with head and neck cancer: a population-based case-control study in Scotland. Br J Oral Maxillofac Surg. 2010;48:11-7.

15. Day GL, Blot WJ, Austin DF, Bernstein L, Greenberg RS, Preston-Martin S, et al. Racial differences in risk of oral and pharyngeal cancer: alcohol, tobacco, and other determinants. J Natl Cancer Inst. 1993;85(6):465-73.

16. Greenberg RS, Haber MJ, Clark WS, Brockman JE, Liff JM, Schoenberg JB, et al. The relation of socioeconomic status to oral and pharyngeal cancer. Epidemiology. 1991;2(3):194-200.

17. Menvielle G, Luce D, Goldberg P, Leclerc A. Smoking, alcohol drinking, occupational exposures and social inequalities in hypopharyngeal and laryngeal cancer. Int J Epidemiol. 2004;33(4):799-806.

18. Zatonski W, Becher H, Lissowska J, Wahrendorf J. Tobacco, alcohol, and diet in the etiology of laryngeal cancer: a population-based case-control study. Cancer Causes Control. 1991;2(1):3-10.

19. Brown LM, Hoover R, Silverman D, Baris D, Hayes R, Swanson GM, et al. Excess incidence of squamous cell esophageal cancer among US Black men: role of social class and other risk factors. Am J Epidemiol. 2001;153(2):114-22.

20. Gammon MD, Schoenberg JB, Ahsan H, Risch HA, Vaughan TL, Chow WH, et al. Tobacco, alcohol, and socioeconomic status and adenocarcinomas of the esophagus and gastric cardia. J Natl Cancer Inst. 1997;89(17):1277-84.

21. Sharp L, Chilvers CE, Cheng KK, McKinney PA, Logan RF, Cook-Mozaffari P, et al. Risk factors for squamous cell carcinoma of the oesophagus in women: a case-control study. Br J Cancer. 2001;85(11):1667-70.
22. Braaten T, Weiderpass E, Kumle M, Lund E. Explaining the socioeconomic variation in cancer risk in the Norwegian Women and Cancer Study. Cancer Epidemiol Biomarkers Preview. 2005;14:2591-7.

23. Heck KE, Pamuk ER. Explaining the relation between education and postmenopausal breast cancer. Am J Epidemiol. 1997;145:366-72.

24. Kropp S, Becher H, Nieters A, Chang-Claude J. Low-to-moderate alcohol consumption and breast cancer risk by age 50 years among women in Germany. Am J Epidemiol. 2001;154(7):624-34.

25. Kruk J. Association of lifestyle and other risk factors with breast cancer according to menopausal status: a case-control study in the Region of Western Pomerania (Poland). Asian Pac J Cancer Prev. 2007:8(4):513-24.

26. Larsen SB, Olsen A, Lynch J, Christensen J, Overvad K, Tjonneland A, et al. Socioeconomic position and lifestyle in relation to breast cancer incidence among postmenopausal women: a prospective cohort study, Denmark, 1993-2006. Cancer Epidemiol. 2011;35(5):438-41.

27. Martin-Moreno JM, Boyle P, Gorgojo L, Willett WC, Gonzalez J, Villar F, et al. Alcoholic beverage consumption and risk of breast cancer in Spain. Cancer Causes Control. 1993;4(4):345-53.

28. Melchior M, Goldberg M, Krieger N, Kawachi I, Menvielle G, Zins M, et al. Occupational class, occupational mobility and cancer incidence among middle-aged men and women: a prospective study of the French GAZEL cohort*. Cancer Causes Control. 2005;16(5):515-24.

29. Robert SA, Strombom I, Trentham-Dietz A, Hampton JM, McElroy JA, Newcomb PA, et al. Socioeconomic risk factors for breast cancer: distinguishing individual- and community-level effects. Epidemiology. 2004;15(4):442-50.

30. Joshi S, Song YM, Kim TH, Cho SI. Socio-economic status and the risk of liver cancer mortality: a prospective study in Korean men. Public Health. 2008;122(11):1144-51.

31. Brown P, Guy M, Broad J. Individual socio-economic status, community socio-economic status and stroke in New Zealand: a case control study. Soc Sci Med. 2005;61(6):1174-88.

32. Kivimaki M, Gimeno D, Ferrie JE, Batty GD, Oksanen T, Jokela M, et al. Socioeconomic position, psychosocial work environment and cerebrovascular disease among women: the Finnish public sector study. Int J Epidemiol. 2009;38(5):1265-71.

33. Kuper $\mathrm{H}$, Adami HO, Weiderpass $E$. The socioeconomic gradient in the incidence of stroke: a prospective study in middle-aged women in Sweden. Stroke. 2007;38:27-33.

34. Laaksonen M, Talala K, Martelin T, Rahkonen O, Roos E, Helakorpi S, et al. Health behaviours as explanations for educational level differences in cardiovascular and all-cause mortality: a follow-up of 60000 men and women over 23 years. Eur J Public Health. 2008;18(1):38-43.

35. McFadden E, Luben R, Wareham N, Bingham S, Khaw K-T. Social class, risk factors, and stroke incidence in men and women: a prospective study in the European prospective investigation into cancer in Norfolk cohort. Stroke. 2009;40(4):1070-7.

36. Sundell L, Salomaa V, Vartiainen E, Poikolainen K, Laatikainen T. Increased stroke risk is related to a binge-drinking habit. Stroke. 2008;39(12):3179-84.

37. Veronesi G, Ferrario MM, Chambless LE, Sega R, Mancia G, Corrao G, et al. Gender differences in the association between education and the incidence of cardiovascular events in Northern Italy. Eur J Public Health. 2011;21(6):762-7.

38. Brummett BH, Babyak MA, Siegler IC, Shanahan M, Harris KM, Elder GH, et al. Systolic blood pressure, socioeconomic status, and biobehavioral risk factors in a nationally representative US young adult sample. Hypertension. 2011;58(2):161-6.

39. Chaix B, Bean K, Leal C, Thomas F, Havard S, Evans D, et al. Individual/ neighborhood social factors and blood pressure in the RECORD Cohort Study: which risk factors explain the associations? Hypertension. 2010;55(3):769-75.

40. Dyer AR, Liu K, Walsh M, Kiefe C, Jacobs Jr DR, Bild DE. Ten-year incidence of elevated blood pressure and its predictors: the CARDIA study. Coronary Artery Risk Development in (Young) Adults. J Hum Hypertens. 1999;13(1):13-21.

41. Radi S, Lang T, Lauwers-Cances V, Diene E, Chatellier G, Larabi L, et al. Job constraints and arterial hypertension: different effects in men and women: the IHPAF II case control study. Occup Environ Med. 2005;62(10):711-7.

42. Mattioli AV, Bonatti S, Zennaro M, Mattioli G. The relationship between personality, socio-economic factors, acute life stress and the development, spontaneous conversion and recurrences of acute lone atrial fibrillation. Europace. 2005;7(3):211-20.

43. Petrovski BÉ, Széles G, Melles M, Pataki V, Kardos L, Jenei T, et al. Behaviour does not fully explain the high risk of chronic liver disease in less educated men in Hungary. Eur J Public Health. 2011;21(5):662-6. 
44. Mäkelä P, Paljärvi T. Do consequences of a given pattern of drinking vary by socioeconomic status? A mortality and hospitalisation follow-up for alcohol-related causes of the Finnish Drinking Habits Surveys. J Epidemiol Community Health. 2008;62:728-33.

45. Bloomfield K, Grittner U, Kramer S, Gmel G. Social inequalities in alcohol consumption and alcohol-related problems in the study countries of the EU concerted action 'Gender, Culture and Alcohol Problems: a Multi-national Study'. Alcohol Alcohol. 2006;41:i26-36.

46. Caldwell TM, Rodgers B, Clark C, Jefferis BJMH, Stansfeld SA, Power C. Lifecourse socioeconomic predictors of midlife drinking patterns, problems and abstention: findings from the 1958 British Birth Cohort Study. Drug Alcohol Depend. 2008;95:269-78.

47. Droomers M, Schrijvers C, Stronks K, van de Mheen D, Mackenbach J. Educational differences in excessive alcohol consumption: the role of psychosocial and material stressors. Prev Med. 1999;29:1-10.

48. Matheson FI, White HL, Moineddin R, Dunn JR, Glazier RH. Drinking in context: the influence of gender and neighbourhood deprivation on alcohol consumption. J Epidemiol Community Health. 2011;66:e4. doi:10.1136/jech.2010.112441

49. Kuipers MAG, Jongeneel-Grimen B, Droomers M, Wingen M, Stronks K, Kunst $A E$. Why residents of Dutch deprived neighbourhoods are less likely to be heavy drinkers: the role of individual and contextual characteristics. J Epidemiol Community Health. 2013;67:587-94.

50. Galea S, Ahern J, Tracy M, Vlahov D. Neighborhood income and income distribution and the use of cigarettes, alcohol and marijuana. Am J Prev Med. 2007;32(6 Suppl):S195-202.

51. Rehm J, Kanteres F, Lachenmeier D. Unrecorded consumption, quality of alcohol and health consequences. Drug Alcohol Rev. 2010;29:426-36.

52. De Boni R, Bertoni N, Bastos L, Bastos F. Unrecorded alcohol in Rio de Janeiro: assessing its misusers through Respondent Driven Sampling. Drug Alcohol Depend. 2014;139:169-73.

53. Hart CL, Davey Smith G, Gruer L, Watt GCM. The combined effect of smoking tobacco and drinking alcohol on cause-specific mortality: a 30 year cohort study. BMC Public Health. 2010;10:789.

54. Mulia N, Schmidt LA, Ye Y, Greenfield TK. Preventing disparities in alcohol screening and brief intervention: the need to move beyond primary care. Alcohol Clin Exp Res. 2011;35:1557-60.

55. Selin KH. Predicting alcohol-related harm by sociodemographic background: high prevalence versus high risk. Contemp Drug Probl. 2005;32:547-88.

56. Huckle T, You RQ, Casswell S. Socio-economic status predicts drinking patterns but not alcohol-related consequences independently. Addiction. 2010;105:1192-202.

57. Mulia N, Karriker-Jaffe KJ. Interactive influences of neighborhood and individual socioeconomic status on alcohol consumption and problems. Alcohol Alcohol. 2012;47:178-86.

\section{Submit your next manuscript to BioMed Central and take full advantage of:}

- Convenient online submission

- Thorough peer review

- No space constraints or color figure charges

- Immediate publication on acceptance

- Inclusion in PubMed, CAS, Scopus and Google Scholar

- Research which is freely available for redistribution

Submit your manuscript at www.biomedcentral.com/submit 